\title{
Optical materials for near infrared Wollaston prisms
}

\author{
E. Oliva ${ }^{1}$, S. Gennari ${ }^{1}$, L. Vanzi ${ }^{2}$, A. Caruso ${ }^{2,3}$, and M. Ciofini ${ }^{3}$ \\ 1 Osservatorio Astrofisico di Arcetri, Largo E. Fermi 5, I-50125 Firenze, Italy \\ 2 Dipartimento di Astronomia e Scienza dell Spazio, Università di Firenze, Largo E. Fermi 5, I-50125 Firenze, Italy \\ 3 Istituto Nazionale di Ottica, Largo E. Fermi 6, I-50125 Firenze, Italy
}

Received May 14; accepted September 16, 1996

\begin{abstract}
The optical characteristics of birefringent materials transmitting in the near IR $(0.9-2.5 \mu \mathrm{m})$ are analyzed. Wollaston prisms with large beam separation and virtually free from lateral chromatism - e.g. with fields of view $>1$ arcmin and image elongation $<0.3^{\prime \prime}-$ could be manufactured using $\mathrm{AgGaS}_{2}$ or $\mathrm{LiYF}_{4}$ (YLF). These materials are used for non linear applications and may therefore find another interesting application in IR focal plane instruments. From the optical point of view the best material is $\mathrm{AgGaS}_{2}$ but YLF may be practically more convenient because of its lower refractive index $(n \sim 1.45)$, better thermo-mechanical properties and lower price.

New measurements of the transparency of Calcite at room and cryogenic temperatures are also presented, these show significant absorption $\left(\simeq 0.4 \mathrm{~cm}^{-1}\right)$ of the ordinary ray already at $\lambda=2 \mu \mathrm{m}$ and very strong bands (absorption coefficient $a>3 \mathrm{~cm}^{-1}$ ) beyond $2.25 \mu \mathrm{m}$. The absorption does not decrease significantly when the crystals is cooled, $\mathrm{CaCO}_{3}$ should not be therefore used in instruments working beyond $2.0 \mu \mathrm{m}$.
\end{abstract}

Key words: instrumentation: miscellaneous instrumentation: polarimeters

\section{Introduction}

Wollaston prisms are extensively used in focal plane astronomical instruments working at visual/ultraviolet wavelengths (e.g. Fosbury et al. 1989). Their main advantage compared to other polarizers/analyzers is that they produce simultaneous images in two perpendicular polarization states, and this minimizes the effect of transparency variations, i.e. the photometric errors propagating into the polarimetric measurement. The use of Wollastons in the IR was so far much more limited though excellent polarization images obtained with this technique can be found in the literature (e.g. Packham et al. 1996). In this paper

Send offprint requests to: E. Oliva we present a study of the optical properties of infrared birefringent crystals aimed at defining convenient materials to manufacture Wollaston prisms with large beam separation and small lateral chromatism.

The second aim of this paper is to determine the long wavelength transmission cutoff of Calcite at cryogenic temperatures. Surprisingly, no such information exists in the literature and, to the best of our knowledge, all available data are still based on the room temperature work of Nysander (1909) who found that $\mathrm{CaCO}_{3}$ becomes opaque to the ordinary ray at $\lambda \simeq 2.2 \mu \mathrm{m}$.

In Sect. 2 we briefly review the basic concepts of Wollaston prisms and analyze the optical performances of birefringent crystals, including several materials recently developed for non-linear applications. In Sect. 3 we present and discuss new measurements of the transmittance of Calcite at cryogenic temperatures. In Sect. 4 we draw our conclusions.

\section{Wollaston prisms and IR birefringent materials}

Figure 1 is a sketch of a Wollaston prism with a schematic ray-tracing. In the first (entrance) prism the "o" light beam vibrating perpendicular to the optic axis has a refractive index $n_{\mathrm{o}}$ while the e-ray has a refractive index $n_{\mathrm{e}}$. At the prisms interface the refraction index of the o-ray changes from $n_{\mathrm{o}}$ to $n_{\mathrm{e}}$ and the opposite occurs for the e-ray, and when exiting the second prism the angle between the two rays is increased further.

For most practical applications the deviation $\delta$ can be considered symmetric around $\theta$ (i.e. $\delta_{\mathrm{o}} \simeq-\delta_{\mathrm{e}}$ ) and the separation between the o and e rays is given by:

$\delta=\left|\delta_{\mathrm{o}}-\delta_{\mathrm{e}}\right| / 2 \simeq \Delta n \tan \alpha$

where $\Delta n=n_{\mathrm{e}}-n_{\mathrm{o}}$ is the birefringence index of the crystal. For astronomical instruments the separation $\delta$ can be most conveniently expressed in sky-projected angles

$\delta^{\prime \prime} \simeq 2063^{\prime \prime} \Delta n \tan \alpha\left(\frac{D_{\mathrm{p}}}{1 \mathrm{~cm}}\right)\left(\frac{D_{\mathrm{tel}}}{1 \mathrm{~m}}\right)^{-1}$ 


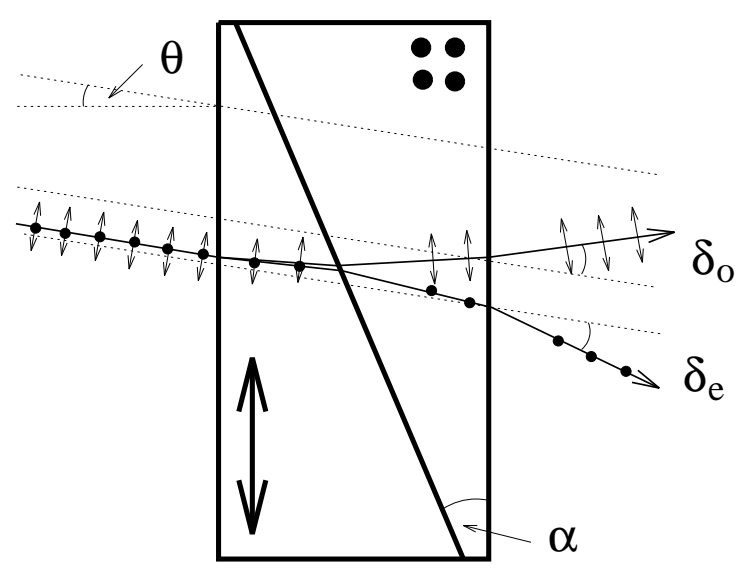

Fig. 1. Schematic representation of a Wollaston prism and ray-tracing (adapted from Fig. 11 of Bennet 1995)

where $D_{\text {tel }}$ is the telescope diameter and $D_{\mathrm{p}}$ is the diameter of the pupil image inside the instrument. The maximum field of view which can be handled by the Wollaston is $2 \delta^{\prime \prime}$. The deviation $\delta$ is chromatic because $\Delta n$ varies with $\lambda$, this implies that broad-band images taken through a Wollaston are elongated. The amount of lateral chromatism, i.e. the image "blurring" over a given $\lambda_{1}<\lambda_{2}<\lambda_{3}$ wavelength range, follows from Eq. (1) and can be conveniently quantified in terms of the parameter

$V=\Delta n\left(\lambda_{2}\right) /\left[\Delta n\left(\lambda_{1}\right)-\Delta n\left(\lambda_{3}\right)\right]$.

The image elongation $\epsilon$ is simply given by

$\epsilon=\delta / V$

and this relationship is independent on the detailed parameters of the prism such e.g. its aperture angle. In the case e.g. of a Calcite Wollaston with a sky-projected separation of $\delta=20^{\prime \prime}$ (i.e. a maximum field of view of $40^{\prime \prime}$ ) the image blurring in the $H$ band is $0.56^{\prime \prime}$ because $V=36$ (cf. Table 1). Wollaston prisms from crystals with $V>100$ could therefore be used to image fields of view $>1$ arcmin with an image blurring of only $<0.3^{\prime \prime}$. These materials are analyzed in some details in the following subsections.

\subsection{AgGaS $S_{2}$ (silver thiogallate)}

This crystal has a quite large birefringence $(|\Delta n|=0.054)$ which peaks at $\simeq 1.05 \mu \mathrm{m}$ and decreases very slowly at longer wavelengths. The combination of large $|\Delta n|$ and low chromatism makes it possible to design Wollaston prisms which can handle large fields of view with subseeing image quality. An example is given in Fig. 2 (left panel) where we plot the computed beam separation for an $\alpha=25^{\circ} \mathrm{AgGaS}_{2}$ Wollaston inserted at the $\oslash 22 \mathrm{~mm}$ pupil plane of NICS, the IR instrument for the Italian $3.5 \mathrm{~m}$ Galileo telescope (Oliva \& Gennari 1995). The separation is $\delta \simeq 32^{\prime \prime}$ and the field of view is therefore 1 arcmin with an image elongation of only $\leq 0.15^{\prime \prime}$ in all bands.

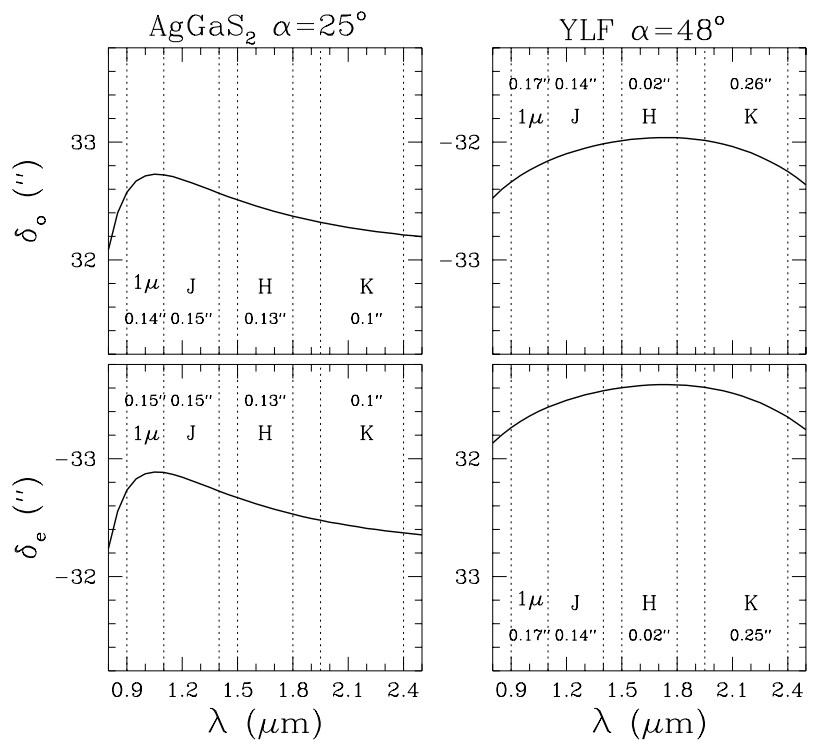

Fig. 2. Predicted separations (sky projected angles) between the "o" and "e" rays in $\mathrm{AgGaS}_{2}$ and $\mathrm{LiYF}_{4}$ (YLF) Wollaston prisms inserted at the $\oslash 22 \mathrm{~mm}$ pupil plane of NICS, the IR instrument for the Italian $3.5 \mathrm{~m}$ Galileo telescope. Note the large total separation of the beams, $\left|\delta_{\mathrm{o}}-\delta_{\mathrm{e}}\right|>1^{\prime}$, and the very small image elongation in the $0.9-1.1, J, H$ and $K$ bands

From the practical point of view, $\mathrm{AgGaS}_{2}$ is considered "one of the most successful materials developed for nonlinear optical laser devices in the infrared" (Bhar et al. 1983). The thermo-optic coefficients are quite large $\left(\mathrm{d} n / \mathrm{d} T \simeq 210^{-4} \mathrm{~K}^{-1}\right)$ but the change of birefringence $\Delta n$ with temperature is small: $\simeq 210^{-6} \mathrm{~K}^{-1}$ (Bhar et al. 1983). No significant change of optical performances is therefore expected when the prism is cooled to $77 \mathrm{~K}$. A potential problem is total reflection at the prisms interface which may be difficult to avoid because of the large refractive index: $n \simeq 2.46$. Standard cements with $n \approx 1.5$ could only be used for prisms with $\alpha \lesssim 30^{\circ}$, and their practical use may be further limited by the fact that $\mathrm{AgGaS}_{2}$ crystals are significantly deformed by cooling. The thermal expansion coefficients $\|$ and $\perp$ to the optical axis are +29 and $-19\left(10^{-6} \mathrm{~K}^{-1}\right)$, respectively.

\section{2. $\mathrm{LiYF}_{4}(Y L F)$}

This material is transparent from below $3000 \AA$ to beyond $4 \mu \mathrm{m}$. It displays a very low chromatism from 1 to $2 \mu \mathrm{m}$ and also has excellent performances from 0.8 to 2.5 $\mu \mathrm{m}$. The computed beam separation for an $\alpha=48^{\circ} \mathrm{YLF}$ Wollaston are displayed in Fig. 2 (right hand panel) where one can better visualize the chromatic performances of this material which is comparable to $\mathrm{AgGaS}_{2}$ in all bands but $K$. The thermo-optics coefficients of YLF are very small, $n_{\mathrm{o}}$ and $n_{\mathrm{e}}$ respectively increase by $1.410^{-4}$ and $5.110^{-4}$ between room temperature and $77 \mathrm{~K}$ (Barnes \& Gettemy 1980). Practical advantages of YLF are: 
Table 1. Refraction indices and chromatic characteristics of IR birefringent crystals with $\left|n_{\mathrm{e}}-n_{\mathrm{o}}\right|>0.01$ (1)

\begin{tabular}{|c|c|c|c|c|c|c|c|c|c|c|}
\hline \multirow[t]{2}{*}{ Material } & \multirow[t]{2}{*}{$n_{\mathrm{O}}^{(2)}$} & \multicolumn{2}{|c|}{$(0.9-1.1)$} & \multicolumn{2}{|c|}{$J(1.1-1.4)$} & \multicolumn{2}{|c|}{$H(1.5-1.8)$} & \multicolumn{2}{|c|}{$K(1.95-2.4)$} & \multirow[t]{2}{*}{$\operatorname{Ref}^{(5)}$} \\
\hline & & $\Delta n^{(3)}$ & $V^{(4)}$ & $\Delta n^{(3)}$ & $V^{(4)}$ & $\Delta n^{(3)}$ & $V^{(4)}$ & $\Delta n^{(3)}$ & $V^{(4)}$ & \\
\hline $\mathrm{Ag}_{3} \mathrm{AsS}_{3}$ & 2.83 & -.237 & 25 & -.229 & 34 & -.223 & 80 & -.220 & 110 & $\mathrm{H} 67$ \\
\hline $\mathbf{A g G a S}_{2}$ & 2.46 & -.054 & -220 & -.054 & 209 & -.054 & 233 & -.054 & 303 & B76 \\
\hline $\mathrm{AgGaSe}_{2}$ & 2.71 & -.022 & -2 & -.029 & -7 & -.031 & -48 & -.032 & -226 & B76 \\
\hline $\mathrm{CaCO}_{3}$ & 1.64 & -.164 & 60 & -.160 & 42 & -.155 & 36 & $-a$ & —a $^{a}$ & B95 \\
\hline $\mathrm{CaWO}_{4}$ & 1.89 & .015 & 34 & .015 & 33 & .014 & 44 & .014 & 35 & B65 \\
\hline $\mathrm{CdS}$ & 2.33 & .018 & 29 & .017 & 30 & .017 & 58 & .016 & 69 & $b$ \\
\hline $\mathrm{CdSe}$ & 2.55 & .020 & -27 & .020 & -233 & .020 & 259 & .020 & 204 & B76 \\
\hline GaSe & 2.92 & -.334 & -15 & -.352 & -26 & -.363 & -75 & -.368 & -130 & P77 \\
\hline$\alpha-\mathrm{HgS}$ & 2.71 & .290 & 22 & .279 & 29 & .271 & 69 & .266 & 101 & B76 \\
\hline $\mathrm{LiIO}_{3}$ & 1.86 & -.140 & 85 & -.138 & 89 & -.137 & 120 & -.135 & 80 & $\mathrm{C} 76$ \\
\hline $\mathrm{LiNbO}_{3}$ & 2.24 & -.079 & 38 & -.077 & 38 & -.075 & 49 & -.072 & 30 & N74 \\
\hline $\mathrm{LiYF}_{4}$ & 1.45 & .022 & 182 & .022 & 223 & .022 & 1290 & .022 & -123 & B80 \\
\hline $\mathrm{MgF}_{2}$ & 1.37 & .012 & 163 & .012 & 114 & .011 & 100 & .011 & 52 & D80 \\
\hline $\mathrm{TiO}_{2}$ & 2.49 & .259 & 41 & .253 & 48 & .248 & 78 & .243 & 55 & B65 \\
\hline $\mathrm{YVO}_{4}$ & 1.96 & .209 & 59 & .206 & 77 & $.203^{c}$ & $173^{c}$ & $.202^{c}$ & $234^{c}$ & $c$ \\
\hline $\mathrm{ZnO}$ & 1.94 & .015 & 42 & .015 & 55 & .014 & 130 & .014 & 220 & B65 \\
\hline
\end{tabular}

(1) The best materials (in bold type) are those with very low lateral chromatism, i.e. $|V|>100$ in all bands.

(2) Refractive index of ordinary ray at $1 \mu \mathrm{m}$.

(3) Birefringence index $\Delta n=n_{\mathrm{e}}-n_{\mathrm{o}}$ at band centers $\left(\lambda_{\mathrm{c}}=1.0,1.25,1.65\right.$ and $\left.2.2 \mu \mathrm{m}\right)$.

(4) Coefficient of lateral chromatism (Eq. 3), the image elongation is proportional to $1 / V$ (Eq. 4).

(5) Reference for the refractive index data.

$a$ Calcite becomes opaque to the ordinary ray at $2.2 \mu \mathrm{m}$, cf. Sect. 3 and Figs. 3, 4.

$b$ Cleveland Crystal Inc. data sheet.

${ }^{c}$ CASIX Inc. data sheet, values beyond $1.4 \mu \mathrm{m}$ may not be accurate, cf. Sect. 2.3.

- The refractive index of $\mathrm{YLF}$ is $\simeq 1.45$ and very close to that of standard optical cements.

- YLF crystals are only slightly deformed by cooling because the thermal expansion coefficients $\|$ and $\perp$ to the crystal axis differ by only $510^{-6} \mathrm{~K}^{-1}$.

- Presently, YLF is much cheaper than $\mathrm{AgGaS}_{2}$.

\subsection{Other materials}

$\mathrm{YVO}_{4}$ is an interesting crystal to which attention was already drawn by Bennet \& Bennet (1978) and which is now produced by several companies. It has a large birefringence $(\Delta n \simeq 0.20)$ and could be therefore useful for manufacturing thin Wollastons when the physical thickness of the prism is limited e.g. by the space available in the filter wheel. Accurate measurement of the refractive indices of $\mathrm{YVO}_{4}$ are not available in the literature and the only information we could find is from the CASIX data sheet which gives the following relationships $(\lambda$ in $\mu \mathrm{m})$

$n_{\mathrm{o}}^{2}=3.77834+.069736 /\left(\lambda^{2}-.04724\right)-.0108133 \lambda^{2}$

$n_{\mathrm{e}}^{2}=4.59905+.110534 /\left(\lambda^{2}-.04813\right)-.0122676 \lambda^{2}$.

These are probably based on measurements at $\lambda<1.4 \mu \mathrm{m}$ and are not necessarily accurate at longer wavelengths.
$\mathrm{MgF}_{2}$ is a well known crystal for quasi-achromatic Wollastons from the ultraviolet to $1 \mu \mathrm{m}$ but has a very low birefringence $(\Delta n=0.011)$ and becomes quite chromatic at $\lambda>2 \mu \mathrm{m}(V=52$ in the $K$ band, cf. Table 1$)$.

CdSe has a very low chromatism in $J, H, K$ but is not suited for applications below $1.1 \mu \mathrm{m}$. Compared to $\mathrm{LiYF}_{4}$ it has a much higher refractive index $(n \simeq 2.55)$ and a slightly lower birefringence $(\Delta n=0.020)$. Both facts make CdSe a not attractive alternative to YLF.

$\mathrm{LiNbO}_{3}$ is a cheap compound extensively used for non linear applications and which is transparent to well beyond $2.5 \mu \mathrm{m}$. It has a quite large birefringence $(|\Delta n| \simeq 0.07)$ and may be useful to manufacture low cost prisms whose performances are however limited by the relatively large chromatism of the crystal $(V \lesssim 40)$.

Rutile $\left(\mathrm{TiO}_{2}\right)$ is another classical material which could be a good though expensive alternative to $\mathrm{LiNbO}_{3}$ whenever high birefringence is required.

Calcite, the most widely used birefringent crystal, has the advantage of low refractive index and high $\Delta n$ but displays a large lateral chromatism and cannot be used beyond $2.0 \mu \mathrm{m}$ because of its large opacity to the ordinary ray (cf. Sect. 3).

$\mathrm{LiIO}_{3}$ has interesting optical properties, large $|\Delta n|$ and $|V| \geq 80$ in all bands, but is very hygroscopic and 


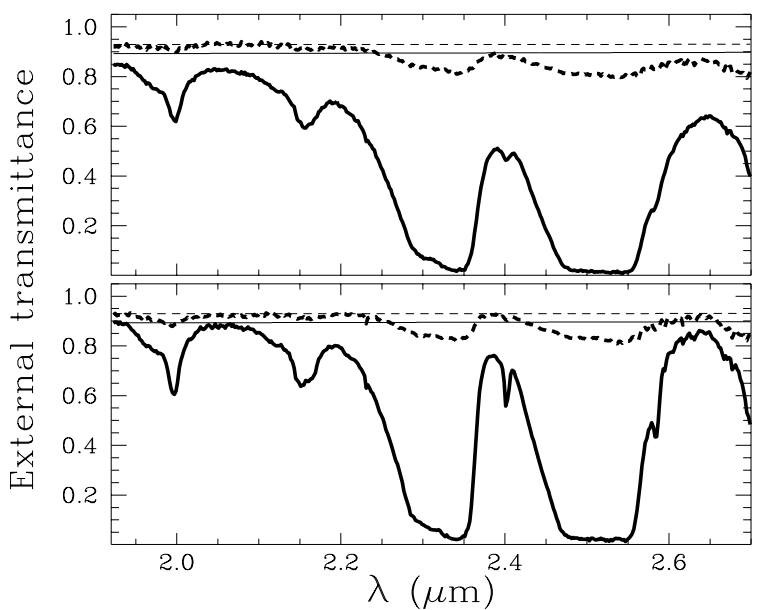

Fig. 3. Transmission of Calcite, thickness $9 \mathrm{~mm}$, at room temperature (upper panel) and $77 \mathrm{~K}$ (lower panel), the solid and dashed lines refer to the ordinary and extraordinary ray, respectively, while the thin lines show the transmission expected including reflection (Fresnel) losses alone

therefore unlikely to find practical applications in astronomical instruments.

\section{Infrared transmittance of Calcite}

$\mathrm{CaCO}_{3}$ is a widely used birefringent material but its use in IR astronomical instruments may be limited by the fact that at room temperatures the crystal becomes opaque to the ordinary ray at $2.2 \mu \mathrm{m}$. The transmittance of $\mathrm{CaCO}_{3}$ at cryogenic temperatures is not known (cf. the Introduction). To fill this gap we performed measurements of Calcite at $20{ }^{\circ} \mathrm{C}$ and $77 \mathrm{~K}$ using the FT-IR PerkinElmer System 2000 spectrophotometer of the National Optical Institute of Florence (Perkins 1986).

The resulting external transmittance is plotted in Fig. 3 where the absorption bands at 2.0, 2.15, 2.35 and $2.5 \mu \mathrm{m}$ are narrower and much deeper than in the old measurements of Nysander (1909) which are reported in optical handbooks. This is also evident in Fig. 4 which compares the absorption coefficients derived here and those of Nysander (1909). Cooling the crystals slightly decreases the absorption at some wavelengths but the overall transmission of our relatively thick $(9 \mathrm{~mm})$ specimen remain quite low.

\section{Conclusions}

Near infrared $(0.9-2.5 \mu \mathrm{m})$ imaging polarimetry of large fields of view ( $>1$ arcmin) with sub-seeing image elongation can be best performed using Wollaston prisms manufactured with $\mathrm{AgGaS}_{2}$ or $\mathrm{LiYF}_{4}$. From the optical point of view the best material is $\mathrm{AgGaS}_{2}$ because it has larger birefringence and smaller chromatism. However, YLF may

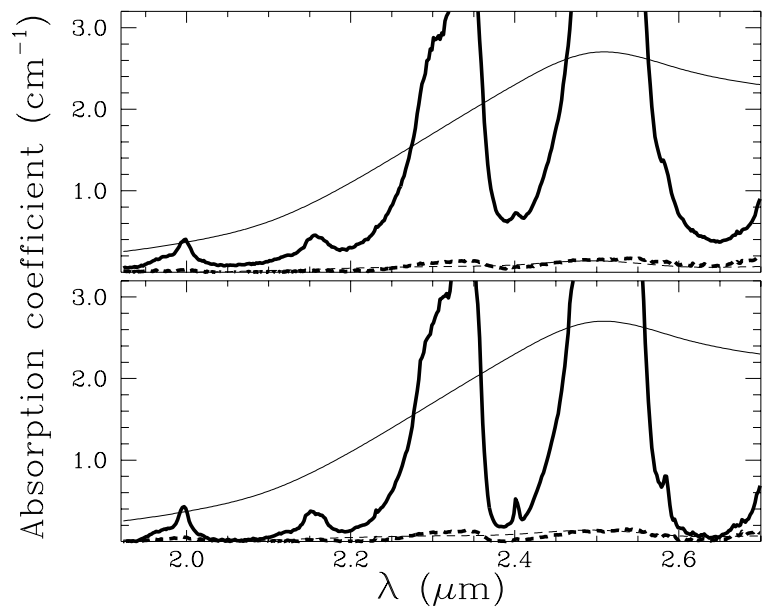

Fig. 4. Absorption coefficient of Calcite for the ordinary (solid lines) and extraordinary (dashed lines) rays at room temperature (upper panel) and $77 \mathrm{~K}$ (lower panel). The thin lines show the data available in optical handbooks (e.g. Bennet 1995) and which are based on the old measurements of Nysader (1909)

be practically more convenient because of its lower refractive index ( $n \sim 1.45$ and similar to optical cements), better thermo-mechanical properties and lower price.

New measurements of the transmittance of Calcite at at $77 \mathrm{~K}$ indicate that $\mathrm{CaCO}_{3}$ cannot be used for applications at wavelengths $\lambda>2 \mu \mathrm{m}$ in astronomical polarimeters.

\section{References}

Bhar G.C., 1976, Appl. Opt. 15, 305 (B76)

Bhar G.C., Ghosh D.K., Ghosh P.S., Schmitt D., 1983, Appl. Opt. 22, 2493

Barnes N.P., Gettemy D.J., 1980, J. Opt. Soc. Am. 70, 1244 (B80)

Bennet H.E., Bennet J.M., 1978, Handbook of Optics $1^{\text {st }}$ edition. In: Driscoll W.G. (ed.). McGraw-Hill, New York, p. $10-63$

Bennet J.M., 1995, Handbook of Optics $2^{\text {nd }}$ edition. In: Bass M. (ed.). McGraw-Hill, New York, Vol. II, Chap. 3 (B95)

Bond W.L., 1965, J. Appl. Phys. 36, 1674 (B65)

Choy M.M., Byer R.L., 1976, Phys. Rev. B 14, 1693 (C76)

Dodge M.J., 1980, Appl. Opt. 23, 1980 (D80)

Fosbury R., Di Serego Alighieri S., Tadhunter C.N., 1989, The Messenger 57, 49

Hulme K.F., Jones O., Davies P.H., Hobden M.V., 1967, Appl. Phys. Lett. 10, 133 (H67)

Nelson D.F., Mikulyak R.M., 1974, J. Appl. Phys. 45, 3688 (N74)

Nysander R.E., 1909, Phys. Rev. 28, 291

Oliva E., Gennari S., 1995, A\&AS 114, 179

Perkins W.D., 1986, J. Chem. Educ. 63, A45

Packham C., Hough J.H., Young S., Chrysostomou A., Bailey J.A., Axon D.J., Ward M.J., 1996, MNRAS 278, 406

Piccioli N., Le Toullec R., Mejatty M., Balkanski M., 1977, Appl. Opt. 16, 1236 (P77) 